\title{
Industrial Challenges for Numerical Simulation of Crystal Growth
}

\author{
Dmitry K. Ofengeim ${ }^{1}$ and Alexander I. Zhmakin ${ }^{1,2}$ \\ 1 Softimpact Ltd., P.O. 83, 194156 St. Petersburg, Russia \\ \{dima, zhmakin\}@softimpact.ru \\ http://www. softimpact.ru/main-engl.html \\ 2 A.F.Ioffe Physical Technical Institute, Russian Academy of Sciences, \\ 194021 St. Petersburg, Russia
}

\begin{abstract}
Numerical simulation of industrial crystal growth is difficult due to its multidisciplinary nature and complex geometry of real-life growth equipment. An attempt is made to itemize physical phenomena dominant in different methods for growth of bulk crystals from melt and from vapour phase and to review corresponding numerical approaches. Academic research and industrial applications are compared. Development of computational engine and graphic user interface of industryoriented codes is discussesd. In conclusion, a simulator for the entire growth process of bulk crystals by sublimation method is described.
\end{abstract}

\section{Introduction}

The aims of numerical simulation are to explain and to predict. Computational Fluid Dynamics (CFD) is certainly one of the most mature computational technologies evolved from academic research into widespread industrial application. However, it is recognized that non-expert is rarely able to apply CFD succesfully to industrial problems 33 . CFD is considered as uncertain discipline and a knowledge-based activity. To cure the situation, EU has launched a huge project QNET-CFD (over 40 organizations from 11 states) with the aim not to perfome actual research, but to assemble and arrange existing knowledge encapsulating CFD use in different industrial sectors (external aerodynamics, combustion, chemical and civil engineering, environment, turbomashinery flows) and to establish best practice guidelines. Numerical simulation of crystal growth, being based on CFD, inherits all its concerns and adds a number of own problems such as unknown phase boundary, facetting of crystal surface, anisotropic crystal properties, radiative heat transfer in the presense of both diffuse and specular reflecting surfaces and semi-transparent bodies, evolution of thermal stresses, point and extended defects during growth as well as during post-process cooling.

In the present paper an attempt is made to review the problems of numerical simulation of industrial crystal growth and approaches to their solution. 


\section{Mathematical Models}

A simulation of the crystal growth requires a solution of a number of subproblems. The key one is the computation of the melt and/or gas flow coupled to the global heat transfer in the growth facility.

Melt Flow. The great variety of melt flow patterns observed in crystal growth systems results from highly different scales of crystals, variation of melt properties (for example, silicon Prandtl number is three order of magnitude smaller that that of some oxides) and numerous driving forces [16].

Bridgman (directional solidification) method is probably the simplest one to simulate. The flow is driven by natural thermal and concentration (double diffusive) convection. The flow is more complex in the case of vertical Bridgman growth with centrifugation 43, being determined by the interaction of buoyancy with Coriolis force. Rotational Bridgman method 30 adds forced convection and free melt/gas surface (and thus Marangoni effect). The forced flow in Czochraski $(\mathrm{Cz})$ growth is caused by the crucible and crystal rotation. An important phenomena to account for is a dynamic gas/melt interaction (the shear stress due to gas flow can greatly exceed the Marangoni stress [17]). In Liquid Encapsulated Czochraski (LEC) method a presence of a third fluid - encapsulant - does not essentially increase the difficulty of the flow computations (encapsulant flow is usually laminar), but could greatly complicate mass transfer problem due to numerous physical-chemical processes in the three media and at interfaces with poorly known rate constants and transport coefficients. In all methods mentioned so far the melt/gas interface can be considered flat while in the floating zone (FZ) method it could be highly curved [28].

One can judge whether the melt flow in the growth system should be laminar or turbulent by the values of the Grashof and (rotational) Reynolds numbers. A winner is surely silicon $\mathrm{Cz}$ growth due to the large size of the crystal and the crucible and the low melt viscosity. Turbulent melt flow in the crucible is non-steady three-dimensional. Axisymmetric approximation could not reproduce experimental data on the melt/crystal phase boundary shape or the oxygen concentration in the crystal which are critical for the crystal quality [18]. Direct Numerical Simulation (DNS) of the melt flow in the large-scale industrial equipment is hardly possible in the near future, to say nothing of the oxygen transport which requires much finer grids for computation due to the high Schmidt number. One should consider with caution the results of so called "coarse DNS" or "quasi-DNS" computations. These approaches does not provide the necessary spatial resolution, but, as their advocates claim, reproduce the flow structures observed experimentally. Indeed, coherent structures in the developed turbulent flow is known to weakly depend on the Reynolds number. However, in the crystal growth problems one is interested first of all in the flow behaviour near the crystal, the crucible and the free surface. At present, the most adequate methods for modelling of the turbulent flow in the industrial growth systems are Large Eddy Simulation (LES) and a hybrid method mixing the best features of LES and an approach based on the Reynolds-averaged Navier-Stokes equations (RANS) [19]. 
Gas Flow. Gas flow in crystal growth governed by the low-Mach number Navier-Stokes equations 23] is laminar in most cases, except high pressure LEC of $A_{3} B_{5}$ crystals. A CFD problem is coupled to mass transfer one for nondilute mixtures [1]. Generally both homogeneous and heterogeneous chemical reactions are to be taken into account, the latter resulting in highly nonlinear boundary conditions.

Radiative Heat Transfer. Due to the high temperatures radiative heat transfer is, as a rule, an important mechanism and frequently a dominant one. It can be even used as a heating method as in FZ method with a double-ellipsoid mirror furnace 22]. The complexity of the adequate radiative transfer model varies greatly. If only opaque solid bodies with diffuse surfaces are present in the growth system, computation of grey radiation using configuration (view) factors ("surface-to-surface") [8] is required. Simulation of growth of semi-transparent crystals is more difficult. One has to account for spectrally dependent absoption in the crystal, specular reflection from the surfaces and different values of the refraction index. Peculiarities of the radiation propagation can cardinally change the shape of the melt/crystal interface in semi-transparent oxides 44]. Thus advanced models, such as characteristics method 34] or extension of Ray Tracing method [25] to multi-band radiative heat transfer [20], are needed.

Phase Boundaries. The simulation of crystal growth from melt includes selfconsistent determination of several interfaces. In $\mathrm{Cz}$ methods boundary melt/gas (melt/encapsulant, encapsulant/gas in LEC) can be usually assumed flat except a small meniscus region. To find this phase boundary in FZ method one had to solve a coupled thermal-electromagnetic-hydrodynamic problem [28].

The boundary melt/crystal is common for all growth methods. One can either track it explicitly or use one of 'uniform' methods such as enthalpy model, level set approach, phase-field model. In the latter, for example, solid phase is considerd as a fluid with very large viscosity 1]. This approach could be optimal for growth of crystals with complex boundaries, such as dendritic solidification [3. In simulation of the industrial growth of single crystal treatment of the melt/crystal boundary as a sharp phase interface is preferable. For solidification of "pure" substance (i.e. when mass diffusion effects can be neglected) process is driven by the temperature alone and described by a classical Stefan problem. In quasi-stationary formulation the interface should be fitted in such a way that the growth rate projection on the crystal pulling direction is constant.

Interface description is more difficult when alloy segregation or facetting of the crystal surface occur. In the latter case melt/crystal boundary does not follow the melting point isotherm, but coinsides with a crystallographic plane. Interface undercooling could be large (for example, up to $20 \mathrm{~K}$ in bismuth germanate growth [10]). It is usually claimed that the facetting is harmful for crystal quality [29] and thus should be avoided by keeping thermal gradient above a critical value. However, recently large nearly perfect crystals of bismuth germanate have been grown with fully facetted solidification front [5]. Numerical treatment of the partially facetted interface has been discussed recently in [0]. 
Electromagnetics. The degree of coupling of the electromagnetic problem to other phenomena varies greatly. Magnetic field used to damp the turbulence fluctuations in $\mathrm{Cz}$ method or to provide a controlled action on the melt flow should be computed self-consistently with the flow or considered given, depending on the value of the magnetic Reynolds number. Radio frequency (RF) heating is only weakly coupled to the thermal problem via temperature-dependent material properties in the sublimation crystal growth. On the other hand, in FZ method it is coupled to the flow that determine the free surface shape and even to the dopant segregation at the growth interface via local electric resistivity [28].

Assessment of Crystal Quality. CFD-like simulations themself could provide the growth rate and composition distributions only. The ultimate aim being the crystal quality, one has also to analyse thermal stresses, point defects and dislocations in the grown crystal and their evolution during post-growth processing.

When the crystal deformation is pure elastic, stresses can be computed for any growth stage independently. Thermoelasicity problem is three-dimensional even for an axisymmetric crystal, except the case of special orientation of the principal crystal axes [27,47]. Stresses caused by the temperature gradients (as well as by the compositional inhomogeneity in ternary compounds induced by segregation) could result in the formation of cracks in the crystals [9. When stress level exceeds a critical value, a plastic creep occurs. Models of dislocations evolution usually exploit the plastic strain rate dependence on the deviatoric stress and dislocations parameters ( the density, the velocity, the Burgers vector) [39] and an equation for the evolution of the dislocation density [24 29|37].

The incorporation of intrinsic point defects into a growing crystal and their evolution are of paramount importance for the silicon crystals. These processes are essentially depend on the melt/crystal interface shape, the ratio of the growth rate to the axial temperature gradient and the presence of impurities, i.e. on peculiarities of the global heat and mass transfer. Simulation of defects in $\mathrm{Si}$ crystals has been reviewed recently in ref. 41.

Mathematical models, as well as numerical methods, used for simulation of crystal growth are essentially the same as in other Computational Continuum Mechanics (CCM) applications (fluid dynamics, electromagnetics, elasticity). The main difference between simulation of the thin film growth and the bulk crystal growth is that in the first case the computational domain can be considered fixed due to the smallness of the epitaxial layer thickness. Numerical study of the bulk crystal growth requires use of either moving grids or regeneration of the grid. The latter approach is attractive when one can exploit quasi-stationary simulation of the growth processes (the characteristic time of the crystal shape changes is large compared to hydrodynamic/thermal time).

Evidently, one has to search for a compromise between a model completness and tractability. Re-phrasing the well-known quotation, the development of the model is finished not when there are no more relevant phenomena to incorporate, but when one can not exlude an effect without compromizing the model. 


\section{Software}

Requirements. The center of numerical simulation of crystal growth is moving from university and academia to industry. There are reasons for that: a great number of available commercial and public-domain generic CFD codes [7]; cheap high power hardware; industry's reluctance to reveal proprietary information to outside consulting partners; in-house operation allows to use simulation routinely in everyday work. Industry needs customized multidisciplinary simulators that hide from the user intricacies of numerical issues and allow the engineer to concentrate on her/his problem 46132936. There are two approaches to development of such growth simulators. One can either "wrap" general purpose code(s) or design a "dedicated" simulator. Somewhat exaggerated differences in requirements for software used in academia and industry are given in Table 1 .

Table 1. Comparison of numerical simulation in academia and industry

\begin{tabular}{lll}
\hline Feature & Academia & Industry \\
\hline Aim & Insight & Optimization \\
Geometry & Simple & Complex \\
Domain & Single or a few blocks & Complete multi-block system \\
Physics & One or a few phenomena & Multidisciplinary \\
Properties & Constant or analytical & Real \\
Platform & Workstation up to mainframe & Workstation or cluster \\
Execution time & Not critical & Up to hours \\
Run-time tuning & Possible & Unacceptable \\
Robustness & Not critical & Mandatory \\
User's experience & Up to high & None up to average \\
Foolproof input & Optional & Mandatory \\
On-line help & Optional & Mandatory \\
Documentation & Optional & Mandatory \\
\hline
\end{tabular}

Optimization of Growth Process and Equipment. The aim of optimization is to increase the crystal size and the uniformity of crystal properties, decrease the number of defects and production costs. Straightforward scaling of the growth equipment with the crystal dimension does not work due to the nonlinearity of the underlying physical phenomena. Optimization of industrial growth of bulk crystals and thin films is now in its infancy while that of growth equipment is still in the prenatal state. A few known examples of process optimization use a small number of control parameters (such as heater(s) power/position in $\mathrm{Cz}$ growth [29] or mass flow rates and susceptor rotation rate in CVD [12]) and thus probably does not encounter ill-posedness of the inverse problems to be solved. When the number of control parameters is large, one is forced to use a regularization of some kind [38] as in the optimization of the crucible design for 
$\mathrm{SiC}$ bulk crystal growth [4]. Probably the most advanced example of the crystal growth optimization is an application of the adjoint method to the solution of the inverse problem for the optimal boundary heat flux distribution in the directional solidification and Bridgman method [45].

High fidelity direct problem solvers are not fast enough, thus one is often forced to use, at least at early optimization stages, "surrogate" models [31]. Such low fidelity models could be physically motivated (reduced spatial dimension of the problem) or derived as black-box models via multivariable approximation (regression methods, neural networks, kriging etc. [14]). Note that in the latter case numerical and experimental data could easily be combined in the optimization process. Parametric geometric modeling, being essentially morphing of a few curves/surfaces, severely restricts the search space. To increase the power of the optimization, topological changes in the system configuration should be allowed. To summarize, there is still a long way to the development of software for optimal design of the entire crystal growth system. Obviously, experience in multidisciplinary optimization should be borrowed from more (computationally) mature industry sectors such as aerospace engineering [2]14].

Could Software be User-Friendly? It is claimed that terms such as "userfriendly" or "easy-to-learn" are ambiguous because they are subjective and thus unverifiable [15]. On the other hand, they can be measured (in relative units) one easily can compare two codes using time needed to master code operation by uninitiated user or time required for specification of geometry and problem parameters. To make a code attractive to the industry user, developers should

1. Use robust algorithms that does not require run-time monitoring and tuning

2. Minimize user actions required for problem specification

3. Use units, variables and control parameters specific for growth method

Ideally, a code should be a black-box one that requires no intervention by the user. The price of the robustness is efficiency. To find a compromise, different forms of adaptivity should be exploited. Grid adaptation to the solution easily can be automated, using as a stopping criteria (in steady problems) either specification of the finest grid size 46] or, more properly (but more tediously), an error estimation [32]. For the most time consuming part - iterative solution of large sparse system of equations - one can use an adaptive polyalgorithm (an ordered set of iterative methods from the fast, but the least robust to the most robust slow one) with automatic method switching [35].

Examples of the item 2 are automatic block detection in the geometry entered by the user and automatic updating geometry and grid regeneration during the growth process (caused by crystal shape evolution, inductor or boat movement).

Complete automation could be extremely difficult. Many wide-spread formats for CAD models do not provide the neibourhoods relations for model entities. Thus, the topology of the model must be reconstructed and this procedure is non-trivial due to the erroneous gaps between the neigbour elements 42 .

To conform to the item 3 only the developers' goodwill is needed. 


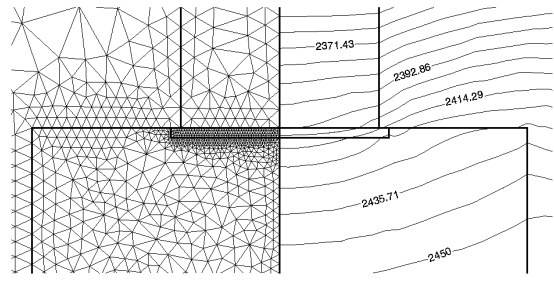

a.

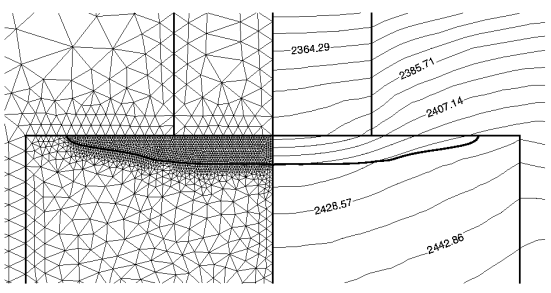

b.

Fig. 1. A fragment of the unstructured nonmatched grid (left) and temperature distribution (right) around crystal at instants: a) $\mathrm{t}=0$; b) $\mathrm{t}=18 \mathrm{~h}$

\section{An Example: A Simulator for Bulk Crystal Growth by Sublimation Method}

In the sublimation method a single crystal is grown from the vapour phase in a closed crucible, the transport being provided by a suitable temperature gradient between the powder charge and the seed.

The code "Virtual Reactor" (VR) [4] for crystal growth by sublimation has been developed as a tool for industry engineers. It provides an accurate solution of all major physical-chemical phenomena relevant to this method such as resistive or RF heating ; conductive, convective and radiative heat transfer; mass transfer in gas and porous media; heterogeneous chemical reaction at catalytic walls and on the surface of powder granules; deposits formation; formation of elastic strain and dislocations in the growing crystal; evolution of crystal and deposit shape. The problem is solved using a quasi-stationary formulation.

Unstructured grid is generated block-wise using Delaune algorithm, an advancing front method or their combination. Non-matched grids in the neighbour blocks are allowed. At each virtual time step a number of subproblems is solved subsequently. Joule heat source distribution is determined by solution of Maxwell equations in the frequency domain. Global heat transfer analysis includes radiative transfer using configuration factors. Darcy law is used to relate the velocity and the pressure distribution in the powder. Conjugate mass transfer in the gas and the powder using Hertz-Knudsen fluxes in the formulation of the boundary conditions for the species concentrations gives the growth rate at the catalitic surfaces. The kind of growing crystal is determined by the type of the surface and local concentration of gas phase components. Computation of thermal stresses and dislocation density is implemented as a post-processing procedure.

Transfer to the next global time instant includes the propagation of the crystal and deposit(s) boundaries, identification of new blocks and boundaries (if needed), the movement of the inductor (if specified by the user) and unstructured grid generation in the new and altered blocks. A special optimization procedure for the growing front advancement has been developed that eliminate the effect of the numerical noise in the growth rate distribution and allows a stable evolution of the crystal and $\operatorname{deposit}(\mathrm{s})$ shape and a monitoring of the topological changes 


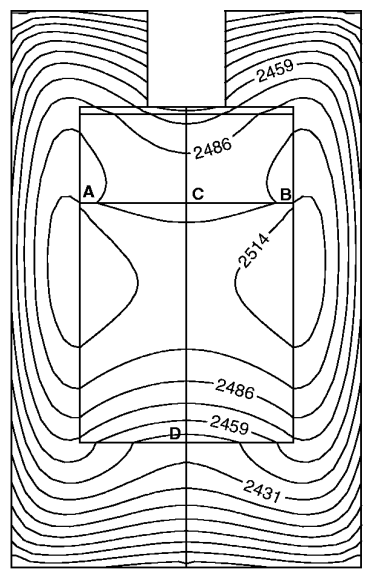

a.

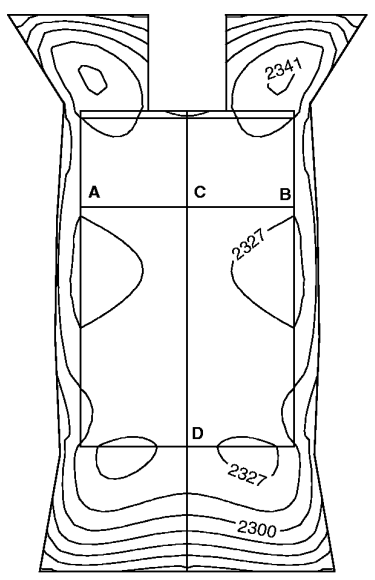

b.

Fig. 2. The crusible shape and isotherms: a) befor optimization; b) after optimization.

in the computational domain. As an example the crystal shape along with the isotherms is shown in Fig. 1 at the growth start and after 18 hours.

VR could be used for the optimization of both crystal growth process and equipment [21]. Fig. 2 illustrate the crucible shape optimization aiming at the temperature uniformity (along $\mathrm{AB}$ and $\mathrm{CD}$ directions) which is known to be favorable for the stable long-term growth.

VR has an easy-to-learn interface that allows the user either to describe the geometry manually or to import a CAD file. All input fields for the problem specification have reasonable default values that allow to start computation directly after the geometry import and the automatic block identification. All geometric and process parameters entered by the user are checked automatically to belong to the corresponding interval of admissible values. The code has a vast extendable data base of material and species properties.

Acknowledgements. The authors are greatful to M.V. Bogdanov, I.Yu. Evstratov, V.V. Kalaev, S.Yu. Karpov, S.K. Kochuguev, A.B. Kulik, Yu.N. Makarov, M.S. Ramm, A.M. Serkov, A.V. Tsirulnikov, I.A. Zhmakin for collaboration and useful discussions.

The work has been supported in part by INTAS Project 00-0263.

\section{References}

1. Anderson, D.M., McFadden, G.B., Wheeler, A.A.: A phase-field model of solidification with convection. Physica D 135 (2000) 175-194

2. Batill, S.V., Renand, J.E., Gu, X.: Modeling and simulation uncertainty in multidisciplinary design optimization. AIAA-2000-4803 (2000) 11 p. 
3. Benes̀, M.: Mathematical and computational aspects of solidification of pure substances. Acta Math. Univ. Comenianae LXX (2001) 123-151

4. Bogdanov, M.V., Galyukov, A.O., Karpov, S.Yu., Kulik, A.V., Kochuguev, S.K., Ofengeim, D.Kh., Tsirulnikov, A.V., Zhmakin, I.A., Komissarov, A.E., Bord, O.V., Ramm, M.S., Zhmakin, A.I., Makarov, Yu.N.: Virtual reactor: a new tool for SiC bulk crystal growth study and optimization. Mat. Sci. Forum, 353-356 (2001) 57-60

5. Borovlev, Yu.A., Ivannikova, N.V., Shlegel, V.N., Vasiliev, Ya.V., Gusev, V.A.: Progress in growth of large sized BGO crystals by the low-thermal-gradient Czochralski technique. J. Crystal Growth 229 (2001) 305-311

6. Cape Simulations Homepage: http://www.capesim.com/simulators.htm

7. CFD codes list: http://www.icemcfd.com/cfd/CFD_codes.html

8. Dupret, F, Nicodeme, P., Ryckmans, Y., Crochet, M.J.: Global modeling of heat transfer in crystal growth furnaces. Int. J. Heat Mass Transfer 33 (1990) 1849-1871

9. Dutta, P.S., Ostrogorski A.G.: Suppression of cracks in $I n_{x} G a_{1-x} S b$ crystals through forced convection in the melt. J. Crystal Growth 194 (1998) 1-7

10. Golyshev, V.D., Gonik, M.A., Tsvetovsky V.B.: In situ measurement of $\mathrm{Bi}_{4} \mathrm{Ge}_{3} \mathrm{O}_{1} 2$ interface supercooling during melt crystal growth. J. Crystal Growth 237-239 (2002) 735-739

11. Egorov, Yu.E., Zhmakin, A.I.: Numerical simulation of low-Mach number gas mixture flows with heat and mass transfer using unstructured grid. Comput. Mater. Sci. 11 (1998) 204-220

12. Eldred, M.S., Hart, W.E., Bohnhoff, W.J., Romero, V.J., Hutchinson, S.A., Salinger A.G.: Utilizing Object-Oriented Design to Build Advanced Optimization Strategies with Generic Implementation. AIAA-96-4164 (1996) 16 p.

13. FEMAG Homepage: http://www.meca.ucl.ac.be/ femag/products.html

14. Giesing, J.P., Barthelemy, J.-F.M.: A Summary of Industry MDO Applications and Needs. AIAA-98-4737 (1998) 20 p.

15. Hooks, I.: Writing Good Requirements. Proc. 3rd Int. Symp. NCOSE (1993) http://www.incose.org/rwg/writing.html

16. Hurle, D.T.J.(ed.): Handbook of Crystal Growth. Bulk Crystal Growth (1994)

17. Kalaev, V.V., Evstratov, I.Yu., Makarov, Yu.N.: Gas flow effect on global heat transport and melt convection in Czochralskii silicon growth. J. Crystal Growth 249 (2003) 87-99

18. Kalaev, V.V., Zhmakin, A.I., Smirnov, E.M.: Modeling of turbulent melt convection during Czochralski bulk crystal growth: J. of Turbulence 3 (2002) 013

19. Kalaev, V.V., Zhmakin A.I.: Large Eddy Simulation of melt convection during Czochralski crystal growth. Proc. 9th Europ. Turbul. Conf. (2002) 207-210

20. Kochuguev, S., Ofengeim, D., Zhmakin, A., Galyukov, A.: Ray tracing method for axisymmetrical global heat transfer simulation. CFD Journal II-33 (2001) 440-448

21. Kulik, A.V., Bogdanov, M.V., Ofengeim, D.Kh., Kochuguev, S.K., Demina, S.E., Karpov, S.Yu., Zhmakin, A.I., Ramm, M.S., Makarov, Yu.N.: Modeling and optimization of $\mathrm{SiC}$ bulk crystal growth by sublimation technique. Proc. 4th Int. Conf. Single Crystal Growth and Heat Mass Transfer (2001) 698-705

22. Lan, C.W.: Three-dimensional simulation of floating-zone crystal growth of oxide crystals. J. Crystal Growth 247 (2003) 597-612

23. Makarov, Yu.N., Zhmakin, A.I.: On flow regimes in VPE reactors. J. Crystal Growth 94 (1989) 537-551

24. Maroudas, D., Brown, R.A.: On the prediction of dislocation formation in semiconductor crystals grown from the melt: analysis of the haasen model for plastic deformation dynamics. J. Cryst. Growth 108 (1991) 399-415 
25. Maruyama, S., Aihira, T.: Radiation heat transfer of arbitrary three-dimensional absorbing,emitting and scattering media and specular and diffuse surfaces. J. Heat Transfer 119 (1997) 129-136

26. Meyappan, M. (ed.): Computational Modeling in Semiconductor Processing (1995)

27. Miyazaki, N.: Development of a thermal stress analysis system for anisotropic single crystal growth. J. Crystal Growth 236 (2002) 455-465

28. Muehlbauer, A., Muiznieks, A., Raming, G.: System of mathematical models for the analysis of industrial FZ-Si-Crystal Growth Processes. Cryst. Res. Technol. 34 (1999) 217-226

29. Müller, G.: Experimental analysis and modeling of melt growth processes. J. Crystal Growth 237-239 (2002) 1628-1637

30. Ozawa, T., Hayakawa, Y., Balakrishnan, K., Kumagawa, M.: Numerical simulation of effect of ampoule rotation for the growth of InGaSb by rotational Bridgman method. J. Crystal Growth 237-239 (2002) 1692-1696

31. Papalambros, P.Y.: Extending the optimization paradigm in engineering design. Proc 3rd Int. Symp. Tools Meth. Compet. Engineer. Delft (2000) 14 p.

32. Prudhomme, S., Oden, J.T., Westermann, T., Bass, J., Botkin, M.E.: Practical Methods for a posteriori Error Estimation in Engineering Applications. Int. J. Num. Meth. Engineer. 56 (2003) 1193-1224

33. Hirsh, Ch.: The QNET-CFD project. Network Bulletin 1 (2001) 4-5. http://www.qnet-cfd.net/newsletter/1st/newsletter-1.pdf

34. Rukolaine, S.A., Vasilyev, M.G., Yuferev, V.S., Galyukov, A.O.: Numerical solution of axisymmetric radiative transfer problems in arbitrary domains using the characteristic method. J. Quant. Spectr. Radiat. Transfer 73 (2002) 205-217

35. Schönauer, W.: Numerical engineering: design of PDE black-box solvers. Math. Comput. Simul. 54 (2000) 269-277

36. Semiconductor Technology Research, Inc. Homepage: http://www.semitech.us/

37. Suezawa, M., Sumino, K., Yonenenaga, N.: Dislocation dynamics in the plastic deformation of silicon crystals. Phys. Stat. Sol. (a) 51 (1979) 217-233

38. Tihonov, A.N., Arsenin, V.Ya.: Solution of Ill-posed problems. New York (1979)

39. Tsai, C.T.: On the finite element modeling of dislocation dynamics during semiconductor crystal growth. J. Cryst. Growth 113 (1991) 499-507

40. Virozub, A., Brandon, S.: Selecting finite element basis functions for computation of partially facetted melt/crystal interfaces appearing during the directional growth of large-scale single crystals. Model. Simul. Mater. Sci. Eng. 10 (2002) 57-72

41. Voronkov, V.V., Falster R.: Intrinsic point defects and impurities in silicon crystal growth. J. Electrochem. Soc. 149 (2002) G167-G174

42. Weihe, K., Willhalm, Th.: Why CAD data repair requires discrete algorithmic techniques. Konstanzer Schrift. Math. Inform. N 61 (1998) 12 p.

43. Wilcox, W.R., Regel, L.L., Arnold, W.A.: Convection and segregation during vertical Bridgman growth with centrifugation. J. Crystal Growth 187 (1998) 543-558

44. Yuferev, V.S., Budenkova, O.N., Vasiliev M.G., et.al. : Variations of solid-liquid interface in BGO low thermal gradients $\mathrm{Cz}$ growth for diffuse and specular crystal side surface. J. Crystal Growth (2003) in print

45. Zabaras, N.: Adjoint methods for inverse free convection problems with applications to solidification processes. Comp. Meth. Opt. Design Control (1998) 391-426

46. Zhmakin, A.I.: A memory-efficient unstructured grid refinement algorithm for computation of 3d steady viscous flows. Comm. Num. Meth. Eng. 13:219-228, 1997.

47. Zhmakin, I.A., Kulik, A.V., Karpov, S.Yu., Demina, S.E., Ramm, M.S.., Makarov, Yu.N.: Evolution of thermoelastic strain and dislocation density during sublimation growth of silicon carbide. Diamond and Related Materials 9 (2000) 446-451 